\title{
Neutron activation cross sections on lead isotopes
}

\author{
V. Semkova, ${ }^{*}$ P. Reimer, T. Altzitzoglou, A. J. M. Plompen, ${ }^{\dagger}$ C. Quétel, S. Sudár, ${ }^{\ddagger}$ and J. Vogl \\ European Commission, Joint Research Centre, Institute for Reference Materials and Measurements, B-2440 Geel, Belgium
}

A. J. Koning

Nuclear Research and Consultancy Group NRG, P. O. Box 25, 1755 ZG Petten, The Netherlands

S. M. Qaim

Institut für Nuklearchemie, Forschungszentrum Jülich GmbH, D-52425 Jülich, Germany

D. L. Smith

Nuclear Engineering Division, Argonne National Laboratory, Argonne, Illinois 60439, USA

(Received 25 September 2008; revised manuscript received 18 June 2009; published 28 August 2009)

\begin{abstract}
The cross sections for the reactions ${ }^{204} \mathrm{~Pb}\left(n, n^{\prime} \gamma\right){ }^{204} \mathrm{~Pb}^{m}, \quad{ }^{204} \mathrm{~Pb}(n, 2 n){ }^{203} \mathrm{~Pb}, \quad{ }^{204} \mathrm{~Pb}(n, 2 n){ }^{203} \mathrm{~Pb}^{m 1}$, $\left.{ }^{204} \mathrm{~Pb}(n, 3 n)\right)^{202} \mathrm{~Pb}^{m},{ }^{206} \mathrm{~Pb}(n, 3 n){ }^{204} \mathrm{~Pb}^{m},{ }^{206} \mathrm{~Pb}(n, \alpha){ }^{203} \mathrm{Hg}$, and ${ }^{208} \mathrm{~Pb}(n, p){ }^{208} \mathrm{Tl}$ were determined at the IRMM van de Graaff laboratory in the neutron energy range from 14 to $21 \mathrm{MeV}$. Both natural and enriched samples were irradiated with neutrons produced via the ${ }^{3} \mathrm{H}(d, n)^{4} \mathrm{He}$ reaction. The induced activities were determined by gamma-ray spectrometry using a HPGe detector in a low-background shield. Neutron fluences were determined with the well-known cross section of the ${ }^{27} \mathrm{Al}(n, \alpha)^{24} \mathrm{Na}$ reaction. Enriched samples were essential to determine the cross sections for the reactions with ${ }^{204} \mathrm{~Pb}^{m}$ and ${ }^{206} \mathrm{~Pb}^{m}$ isomers in the final state. Accurate results for reactions with ${ }^{204,206} \mathrm{~Pb}$ as target nuclei with natural lead samples were enabled through a precise measurement of the isotopic ratios. For a first investigation of the consequences of the present data for nuclear reaction models they were confronted with calculations based on global parameter systematics in a phenomenological and in a microscopic approach and with parameters selected to reproduce the available data. The TALYS code was used for the former two calculations involving parameter systematics while the STAPRE code was used for the latter calculation.
\end{abstract}

DOI: 10.1103/PhysRevC.80.024610

PACS number(s): 25.40.-h, 21.10.Ma, 24.10.-i, 28.20.-v

\section{INTRODUCTION}

The isotopes of lead were studied extensively both experimentally and theoretically on account of the double shell closure of ${ }^{208} \mathrm{~Pb}$ and the large number of isotopes that allow experimental nuclear structure studies for the $Z=82$ closed shell. In addition, scattering off the stable nuclides was studied extensively both experimentally and theoretically to test ideas concerning the interaction of nucleons [1] and complex light particles with nuclei.

In comparison, relatively little is known about neutroninduced reactions above $100 \mathrm{keV}$. In this work, we have obtained new results for one inelastic scattering, two $(n, 2 n)$, two $(n, 3 n)$, one $(n, p)$, and one $(n, \alpha)$ reaction on isotopes of lead using the activation technique. To enable this study, enriched lead samples were used. Results were obtained for the first time for the reactions ${ }^{204} \mathrm{~Pb}(n, 3 n){ }^{202} \mathrm{~Pb}^{m},{ }^{204} \mathrm{~Pb}(n, 2 n){ }^{203} \mathrm{~Pb}^{m 1}$, ${ }^{206} \mathrm{~Pb}(n, 3 n)^{204} \mathrm{~Pb}^{m}$ and ${ }^{206} \mathrm{~Pb}(n, \alpha){ }^{203} \mathrm{Hg}$ in the energy range studied, whereas important complementary results were

\footnotetext{
*Present address: Institute for Nuclear Research and Nuclear Energy, Bulgarian Academy of Sciences, 1784 Sofia, Bulgaria.

${ }^{\dagger}$ Arjan.Plompen@ec.europa.eu

${ }^{\ddagger}$ Present address: Institute for Experimental Physics, University of Debrecen, H-4001 Hungary.
}

obtained for the ${ }^{204} \mathrm{~Pb}\left(n, n^{\prime} \gamma\right){ }^{204} \mathrm{~Pb}^{m},{ }^{204} \mathrm{~Pb}(n, 2 n){ }^{203} \mathrm{~Pb}$, and ${ }^{208} \mathrm{~Pb}(n, p){ }^{208} \mathrm{Tl}$ reactions.

The data are compared with three state-of-the-art model calculations performed with two different model codes. Two of these calculations are based on parameter systematics that were obtained by a global adjustment to the available data across the mass table. In one case this concerns a purely phenomenological model while in the other case optical model, level density and gamma-ray strength functions were obtained from microscopic models. The comparison with the present experimental results allows to judge the predictive power of these models. In a third calculation parameters of a phenomenological model were selected carefully based on all available experimental information including that presented here. Thus, some additional insight is provided when global systematics does not result in quantitative agreement with the data.

Four of the results concern cross sections for the isomers $\left({ }^{202} \mathrm{~Pb}^{m},{ }^{203} \mathrm{~Pb}^{m 1},{ }^{204} \mathrm{~Pb}^{m}\right)$ populated through $(n, x n \gamma)$ reactions with $x=1-3\left({ }^{204} \mathrm{~Pb}(n, 3 n){ }^{202} \mathrm{~Pb}^{m},{ }^{204} \mathrm{~Pb}(n, 2 n){ }^{203} \mathrm{~Pb}^{m 1}\right.$, $\left.{ }^{204} \mathrm{~Pb}\left(n, n^{\prime} \gamma\right){ }^{204} \mathrm{~Pb}^{m},{ }^{206} \mathrm{~Pb}(n, 3 n){ }^{204} \mathrm{~Pb}^{m}\right)$. These are valuable as they provide additional information about the reaction mechanism, i.e., they are sensitive to the decay scheme, the spin and parity dependence of the level density and the initial population of the levels that feed the isomers. In this respect, isomer cross sections are very similar to $(n, x n \gamma)$ cross sections 
obtained for states that decay with much shorter half-lives. Related earlier work of the present authors includes the study of such $(n, x n \gamma)$ cross sections for lead isotopes and bismuth and the population of isomers of $\mathrm{Au}$ and $\mathrm{Hg}$ through different reaction channels [2-5].

Recently, the study of neutron-induced reactions has received considerable attention, as lead or lead-bismuth eutectic is considered as a possible coolant and/or spallation target for certain advanced nuclear reactors. A pertinent recent example of such a study may be found in Ref. [6], in which one also finds further references.

\section{EXPERIMENTS}

The ${ }^{204} \mathrm{~Pb}\left(n, n^{\prime} \gamma\right){ }^{204} \mathrm{~Pb}^{m}, \quad{ }^{204} \mathrm{~Pb}(n, 2 n){ }^{203} \mathrm{~Pb}, \quad{ }^{204} \mathrm{~Pb}(n$, $2 n)^{203} \mathrm{~Pb}^{m 1},{ }^{204} \mathrm{~Pb}(n, 3 n){ }^{202} \mathrm{~Pb}^{m},{ }^{206} \mathrm{~Pb}(n, 3 n){ }^{204} \mathrm{~Pb}^{m},{ }^{206} \mathrm{~Pb}(n$, $\alpha)^{203} \mathrm{Hg}$, and ${ }^{208} \mathrm{~Pb}(n, p){ }^{208} \mathrm{Tl}$ reactions were studied with quasi-monoenergetic neutrons at the IRMM Van de Graaff accelerator laboratory using the activation technique. The activities were determined by gamma-ray counting with high purity germanium (HPGe) detectors. In the case of ${ }^{203} \mathrm{~Pb}^{m}$, the very short half-life required a fast pneumatic transport system to iterate between irradiation and counting. The methods employed here closely follow those of our earlier works [7-13]. Here, we concentrate on the features relevant for the present work.

\section{A. Samples}

Both isotopically enriched samples and materials of natural abundance were employed in the present measurements. The natural lead was supplied as metal rods by Advent Research Materials Ltd., Oakfield Industrial Estate, Eynsham-Oxon, England OX8 1JA. Special attention was paid to the purity of the sample to minimise interferences. The lead rods were rolled down to a thickness of $0.5-0.6 \mathrm{~mm}$ and then punched to disks of $13 \mathrm{~mm}$ diameter. Stacks of one to 12 such disks were irradiated depending on the expected count rate.

Since the composition of natural lead varies [14] depending on origin and age [15], an isotopic analysis of the sample material was performed by the IRMM Isotopic Measurement Unit. A comparison of the obtained results is shown in Table I together with the representative values. It is evident that the isotopic analysis allows measurement results with an overall uncertainty well below $10 \%$ for measurements on the target nuclei ${ }^{204} \mathrm{~Pb}$ and ${ }^{206} \mathrm{~Pb}$, whereas the natural lead used here shows a lower abundance for ${ }^{204} \mathrm{~Pb}$ by $8.3 \%$ and a higher abundance by $13 \%$ for ${ }^{206} \mathrm{~Pb}$ than the respective representative values. These deviations are well out of the range of the standard spread on these representative values, but within the much larger range of natural variation.

The enriched ${ }^{206} \mathrm{~Pb}$ was supplied by Chemotrade Chemiehandels-gesell-schaft mbH, 40239 Düsseldorf, Germany. About $100 \mathrm{mg}$ of metal was centered in a cardboard ring of $13 \mathrm{~mm}$ outer diameter and $5 \mathrm{~mm}$ inner diameter and fixed with adhesive tape. A $219.3 \mathrm{mg}$ sample of metallic lead enriched to $48.9 \%{ }^{204} \mathrm{~Pb}$ was supplied by JSC “JV ISOFLEX," Moscow, Russia. This sample pressed into a pellet of $6 \mathrm{~mm}$ diameter and $0.8 \mathrm{~mm}$ thickness was also centered in a cardboard ring of $13 \mathrm{~mm}$ outer diameter and fixed with adhesive tape. $\mathrm{Al}, \mathrm{Fe}, \mathrm{Nb}$, $\mathrm{Ni}$, and In high-purity metal foils supplied by Goodfellow Metals, Cambridge, UK, were used as neutron fluence monitors. The monitors were disk-shaped with 50-300 $\mu \mathrm{m}$ thickness and $13 \mathrm{~mm}$ diameter.

\section{B. Irradiations}

The irradiations were carried out at the $7 \mathrm{MV}$ Van de Graaff accelerator. Quasi-monoenergetic neutrons with energies between 14.8 and $20.5 \mathrm{MeV}$ were produced via the ${ }^{3} \mathrm{H}(d, n){ }^{4} \mathrm{He}$ reaction $(Q=17.59 \mathrm{MeV})$ using a solid-state $\mathrm{Ti} / \mathrm{T}$ target of $2 \mathrm{mg} / \mathrm{cm}^{2}$ thickness on a silver backing of $0.4 \mathrm{~mm}$ thickness. The incident deuteron energies were 1,2 , 3 , and $4 \mathrm{MeV}$. Neutrons with energy $3.45 \mathrm{MeV}$ were produced by a $4.3 \mathrm{MeV}$ proton beam on a solid-state Ti/T target via the ${ }^{3} \mathrm{H}(p, n){ }^{3} \mathrm{He}$ reaction $(Q=-0.764 \mathrm{MeV})$. The typical beam current was $10 \mu \mathrm{A}$. The energy scale of the accelerator was calibrated with an uncertainty of about $5 \mathrm{keV}$. The standard spread of the neutron energy distribution ranged from 30 to $250 \mathrm{keV}$ as a result of energy loss by the projectile in the target and the variation of the energy of the neutron with the angle (see also, Tables IV and V). Typical combined uncertainties of the mean neutron energy were $35,15,10$, and $10 \mathrm{keV}$ at deuteron energies of $1,2,3$, and $4 \mathrm{MeV}$ (zero degrees, $\sim 1.5 \mathrm{~cm}$ ). A long counter operating in a multichannel scaling acquisition mode was used to record the time profile of the neutron flux during all irradiations.

Two different irradiation setups were used, depending on the half-life of the reaction product. The first was a light weight Al holder that allowed samples to be placed at a range of angles and distances. Here it was employed for activities with half-lives larger than 3 min using angles from 0 to $75^{\circ}$ and distances from 1 to $4 \mathrm{~cm}$.

TABLE I. Isotopic composition of different lead samples (in \%).

\begin{tabular}{|c|c|c|c|c|}
\hline & ${ }^{204} \mathrm{~Pb}$ & ${ }^{206} \mathrm{~Pb}$ & ${ }^{207} \mathrm{~Pb}$ & ${ }^{208} \mathrm{~Pb}$ \\
\hline Range of natural variation ${ }^{a}$ & $1.65-1.04$ & $27.48-20.84$ & $23.65-17.62$ & $56.21-51.28$ \\
\hline Representative values $^{\mathrm{a}}$ & $1.4(1)$ & $24.1(1)$ & $22.1(1)$ & $52.4(1)$ \\
\hline Nat. sample, this work ${ }^{b}$ & $1.284(20)$ & $27.12(26)$ & $20.49(17)$ & $51.11(24)$ \\
\hline Enriched ${ }^{204} \mathrm{~Pb}^{\mathrm{c}}$ & 48.7 & 6.2 & 7.2 & 37.9 \\
\hline Enriched ${ }^{206} \mathrm{~Pb}^{\mathrm{c}}$ & - & 94.00 & 4.04 & 1.96 \\
\hline
\end{tabular}

\footnotetext{
${ }^{\text {aTTaken from [14]. }}$

${ }^{\mathrm{b}}$ Measured with ICP-MS, this work.

${ }^{\mathrm{c}}$ Given by the supplier.
} 
For the study of the ${ }^{204} \mathrm{~Pb}(n, 2 n)^{203} \mathrm{~Pb}^{m 1}$ reaction a pneumatic transport system was used to automatically iterate the irradiation and activity determination with a natural lead sample until sufficient statistics were acquired and to limit the time between irradiation and counting to 4.0(1) s. One disk of natural lead was irradiated at $0^{\circ}$ relative to the incident deuteron beam and the distance between the sample and back of the target was $16 \mathrm{~mm}$. In each cycle the sample was irradiated for five half-lives while its activity was counted for three half-lives. The sample transport and data acquisition were controlled by the DAQ2000 system developed at IRMM. To obtain an accurate determination of the cooling time, this system records both the neutron flux time profile measured by the long-counter and the gamma-spectra from the HPGe detector. A multichannel scaler is used to register for the whole measurement (all cycles) the neutron-flux time-profile, the time between end of irradiation and start of counting, the counting period and the period from the end of counting to the start of irradiation with a resolution of $0.1 \mathrm{~s}$. To this end two signals are input via a logic-OR: the counts observed by a long-counter that monitors the neutron flux time profile and an acquisition enabled pulser.

For the ${ }^{208} \mathrm{~Pb}(n, p)^{208} \mathrm{Tl}$ reaction, cross sections were deduced from the above mentioned irradiations with the pneumatic transport system for the study of the ${ }^{204} \mathrm{~Pb}(n, 2 n)^{203} \mathrm{~Pb}^{m 1}$ reaction. To check that the buildup of ${ }^{208} \mathrm{Tl}$ activity in subsequent irradiations was adequately accounted for, a control measurement was made with the light weight $\mathrm{Al}$ holder at $E_{n}=19.3 \mathrm{MeV}\left(\Theta=0^{\circ}\right)$. The latter setup was also used to obtain the result at $E_{n}=14.8 \mathrm{MeV}\left(\Theta=75^{\circ}, E_{d}=1 \mathrm{MeV}\right)$.

In case the lightweight $\mathrm{Al}$ holder was used, symmetric sample stacks were irradiated consisting of a set of monitor foils ( $\mathrm{Al}, \mathrm{Fe}, \mathrm{Nb}, \mathrm{Ni}, \mathrm{In})$ facing the beam, the sample under study (in the middle) and then another stack of monitor foils at the back of the sample. For thicker stacks $(>4 \mathrm{~Pb}$ disks) a third set of monitor foils was inserted in the middle to better control the flux gradient. For the measurements with the pneumatic transport system sets of monitor foils were irradiated independent of the sample to obtain sufficient activity and to limit the background for the determination of the activity of the sample. The long-counter readings were used to relate the two measurements (see also Ref. [11]).

\section{Mean neutron energies and flux densities}

Mean neutron energies and standard spreads of the primary neutrons incident on a sample were calculated both by the EnergySet [16] program that is based on the reaction tables of Ref. [17] and the stopping powers of Ref. [18] and by the Monte Carlo code TARGET [19], which simulates the neutron production, transport and scattering processes in the target and integrates over the sample volume. These two estimates agree closely.

Above a deuteron energy of $1 \mathrm{MeV}$ the DT neutron spectrum from solid tritium targets shows the presence of low-energy secondary neutrons produced by deuteron reactions with target backing, implanted deuterium from earlier irradiations, and the ${ }^{3} \mathrm{H}(d, n p)$ deuteron-breakup process. The intensity of these secondary neutrons increases strongly with deuteron energy, and furthermore depends on emission angle and target irradiation history. The various components of the neutron flux density spectra were determined by the time-of-flight method for both unused and heavily irradiated targets and the relative intensities of the components were fixed for each irradiation by a spectral index method that involves the above-mentioned monitor reactions and uses the distinct energy threshold and excitation function of a reaction $[11,32]$. The reactions used for the unfolding, the decay data of the reaction products [20], and the references of the required evaluated cross sections are included in Table II. The neutron fluence rate was determined using the well-known ${ }^{27} \mathrm{Al}(n, \alpha)^{24} \mathrm{Na}$ reaction cross section, except for the neutron

TABLE II. Decay data from [20] unless indicated otherwise and references for the cross sections of the neutron fluence monitor reactions. $Q$-values were calculated with QTOOL [21] that is based on the masses given by Audi and Wapstra [22].

\begin{tabular}{lccccc}
\hline \hline Nuclear reaction & Half-life of product & $Q$-value $(\mathrm{MeV})$ & $E_{\gamma}(\mathrm{keV})$ & Intensity (\%) & Ref. \\
\hline \multicolumn{5}{c}{ Reactions studied } \\
${ }^{204} \mathrm{~Pb}(n, 3 n)^{202} \mathrm{~Pb}^{m}$ & $3.53(1) \mathrm{h}$ & -17.488 & 960.7 & $89.9(5)$ & {$[23]$} \\
${ }^{204} \mathrm{~Pb}(n, 2 n)^{203} \mathrm{~Pb}^{204} \mathrm{~Pb}(n, 2 n)^{203} \mathrm{~Pb}^{m 1}$ & $51.92(3) \mathrm{h}$ & -8.394 & 279.2 & $80.9(19)$ & {$[24]$} \\
${ }^{204} \mathrm{~Pb}\left(n, n^{\prime} \gamma\right)^{204} \mathrm{~Pb}^{m}$ & $6.21(8) \mathrm{s}$ & -9.219 & 825.3 & $71.6(7)$ & {$[24]$} \\
${ }^{206} \mathrm{~Pb}(n, 3 n)^{204} \mathrm{~Pb}^{m}$ & $67.2(3) \mathrm{min}$ & -2.185 & 899.2 & $99.17(2)$ & {$[25]$} \\
${ }^{206} \mathrm{~Pb}(n, \alpha)^{203} \mathrm{Hg}$ & $46.594(12) \mathrm{d}$ & -17.005 & 911.7 & $90.69(10)$ & {$[25]$} \\
${ }^{208} \mathrm{~Pb}(n, p)^{208} \mathrm{Tl}$ & $3.053(4) \mathrm{min}$ & -4.129 & 279.2 & $81.56(5)$ & {$[24]$} \\
& & & 583.2 & $85.0(3)$ & {$[26]$} \\
${ }^{115} \mathrm{In}\left(n, n^{\prime} \gamma\right)^{115} \mathrm{In}^{m}$ & $4.486(4) \mathrm{h}$ & -0.336 & 336.2 & $45.8(22)$ & {$[27]$} \\
${ }^{58} \mathrm{Ni}(n, p)^{58} \mathrm{Co}^{27} \mathrm{Al}(n, p)^{27} \mathrm{Mg}$ & $70.86(7) \mathrm{d}$ & 0.401 & 810.8 & $99.45(1)$ & {$[28]$} \\
${ }^{56} \mathrm{Fe}(n, p)^{56} \mathrm{Mn}$ & $9.458(12) \mathrm{min}$ & -1.828 & 843.8 & $71.8(4)$ & {$[28]$} \\
${ }^{27} \mathrm{Al}(n, \alpha)^{24} \mathrm{Na}$ & $2.5789(1) \mathrm{h}$ & -2.913 & 846.8 & $98.9(3)$ & {$[28]$} \\
${ }^{93} \mathrm{Nb}(n, 2 n)^{92} \mathrm{Nb}^{m}$ & $14.997(12) \mathrm{h}$ & -3.133 & 1368.6 & $99.994(2)$ & {$[29]$} \\
\hline \hline
\end{tabular}


energy of $3.45 \mathrm{MeV}$ for which the ${ }^{115} \operatorname{In}\left(n, n^{\prime} \gamma\right)^{115} \operatorname{In}^{m}$ and ${ }^{58} \mathrm{Ni}(n, p){ }^{58} \mathrm{Co}$ dosimetry reactions were used.

\section{Measurement of radioactivity}

Standard $\gamma$-ray spectroscopy was employed for the measurement of radioactivity. Three lead-shielded HPGe detectors were used for the measurements. The mode of operation, measurement and calibration procedures, and the analysis software have been described recently $[7,8]$. The decay data (Table II) were taken from Ref. [20]. For the activity determination, half-lives, emission probability, gamma-ray attenuation, the spatial dependence of the gamma-ray efficiency, and coincidence summing corrections were taken into account. In the case of ${ }^{202} \mathrm{~Pb}^{m},{ }^{203} \mathrm{~Pb}^{g}$, and ${ }^{204} \mathrm{~Pb}^{m}$ the summing corrections were checked experimentally by varying the distance between the detector and the sample and for the remaining radionuclides, calculated values were used based on measured total efficiencies and the available decay schemes [20]. Self-attenuation of $\gamma$-ray s was limited by distributing the lead disks over the detector. The determination of the ${ }^{208} \mathrm{Tl}$ activity induced by many repetitive short irradiations needed accounting for all irradiation cycles prior to each counting period.

\section{E. Processing count rates into cross sections}

The cross sections were calculated using the well-known activation formula, closely following the procedures detailed in Refs. $[7,8,11,12]$. The cross sections for both the ${ }^{204} \mathrm{~Pb}\left(n, n^{\prime} \gamma\right)^{204} \mathrm{~Pb}^{m}$ and ${ }^{206} \mathrm{~Pb}(n, 3 n)^{204} \mathrm{~Pb}^{m}$ reactions leading to the same product radionuclide were determined using enriched ${ }^{204} \mathrm{~Pb}$ and ${ }^{206} \mathrm{~Pb}$ samples following the method described in Refs. [11,12].

Counting rates were corrected for coincidence losses, $\gamma$-ray emission probability, $\gamma$-ray self-absorption, efficiency of the detector, time-dependence of the neutron fluence, and low-energy neutron background. The spectral index method [32] revealed that large corrections were required for the ${ }^{204} \mathrm{~Pb}\left(n, n^{\prime} \gamma\right)^{204} \mathrm{~Pb}^{m}$ reaction cross section at the highest neutron energies studied, as a consequence of the low-energy neutron background. Similar results were found earlier for the ${ }^{89} \mathrm{Y}\left(n, n^{\prime} \gamma\right){ }^{89} \mathrm{Y}^{m}$ [33] and ${ }^{99} \mathrm{Tc}\left(n, n^{\prime} \gamma\right){ }^{99} \mathrm{Tc}^{m}$ [34] reactions. Corrections for the remaining reactions were negligible due to the relatively high effective reaction threshold. The contribution of neutron scattering to the background was corrected for by using the Monte Carlo method [35]. These corrections were the largest at $4 \mathrm{MeV}$ incident deuteron energy, e.g., $11 \%$ for ${ }^{115} \operatorname{In}\left(n, n^{\prime} \gamma\right)^{115} \operatorname{In}^{m}$ reaction, $7 \%$ for ${ }^{58} \mathrm{Ni}(n, p){ }^{58} \mathrm{Co}$ and ${ }^{204} \mathrm{~Pb}\left(n, n^{\prime} \gamma\right){ }^{204} \mathrm{~Pb}^{m}$ reactions, and less than $2 \%$ for all the remaining reactions.

\section{NUCLEAR MODEL CALCULATIONS}

To provide for a first investigation of the physical implications of the data, these were compared with model calculations based on recent systematics and on a model calculation that was adjusted to the data. In all, three calculations are shown below. They were performed with the well-known TALYS [36] and STAPRE [37] codes that use the Hauser-Feshbach model extended with estimates for width fluctuations, the direct interaction, and pre-equilibrium emission. Both codes rely on the Reference Input Parameter Library [38] for many of their parameters. The first calculation, labeled "TALYS," concerns a purely phenomenological model with globally optimized parameters. The second, labeled "TALYS- $\mu$ " concerns a model in which the important quantities were derived from globally optimized microscopic calculations. The third, labeled "STAPRE" concerns a purely phenomenological model, similar to that of TALYS but with parameters optimized to describe the experimental results. The STAPRE code has been used extensively in investigations concerning isomer cross sections [4,33,39-41]. Further details are given below.

\section{A. Calculations with the TALYS code}

Nuclear model calculations were performed with the recent TALYS code, version $1.04[36,42]$. The two calculations may be specified as follows.

\section{TALYS}

The neutron (and proton) spherical optical model is that of Koning and Delaroche [43]. For ${ }^{208} \mathrm{~Pb}$ the same modifications as those used in Ref. [3] were employed for the neutron optical model as these improve the description of scattering data $[3,44]$. The level density model is a combination of the constant temperature approach at low energy and the back-shifted Fermi-gas model with energy dependent level density parameters according to Ignatyuk's proposal. A comprehensive description of the level density model and the method for obtaining the parameters is presented in Ref. [45]. For the present study, this means that for all target and residual nuclides the constant temperature model is automatically adjusted to the known discrete levels, while the Fermi gas part is adjusted to the experimental mean neutron resonance spacing at the neutron separation energy, if available. The two-component exciton model of Ref. [46] is used for the description of pre-equilibrium emission and the distorted wave Born approximation for the direct interaction [36]. Gamma-ray transmission coefficients are obtained from $\gamma$-ray strength functions following Ref. [47] for $E 1$ radiation, and Refs. [48,49], for all other transitions. These transmission coefficients are adjusted to reproduce the average radiative capture width [50]. For the first 25 excited levels in the target and residual nuclei, TALYS relies on a nuclear structure and decay table to describe the de-excitation of the nuclei. This table is derived from Ref. [38] which in turn relies on the ENSDF evaluation [51] current at the time that [38] appeared. The standard value of 20 levels was modified to include the ${ }^{204} \mathrm{~Pb}^{m}$ isomer which is the 21 st excited level of ${ }^{204} \mathrm{~Pb}$.

\section{TALYS- $\mu$}

The neutron and proton optical model is that of Bauge and coworkers [52,53] which in turn is based on nuclear densities, the optical model potential for nucleons in nuclear matter and the requirement of Lane consistency and a global optimisation to measured total and scattering cross sections. 
The combinatorial model for level densities of Ref. [54] was employed. It makes consistent use of nuclear structure properties that are determined by Skyrme-Hartree-Fock-Bogoliubov calculations and is applicable for 8500 nuclei up to $200 \mathrm{MeV}$ excitation energy and spin 49. For further details see Ref. [45] but note that here these densities were not adjusted to reproduce experimental $s$-wave level spacings or the discrete states at low energy. The $\gamma$-ray strength functions are derived from a Hartree-Fock-Bogoliubov approach [38] by Goriely and coworkers. Pre-equilibrium emission is treated in exactly the same way as for the phenomenological TALYS calculation.

The comparisons of the new measured cross sections with the TALYS calculations may be considered as "blind comparisons", since no match to the present data was attempted here. TALYS may also be used to optimally reproduce the experiment and this has been demonstrated for the lead isotopes in Refs. [6,44]. The present comparison with the data is expected to be favorable since important data on lead featured in the global parameter searches mentioned above.

\section{B. Calculations with the STAPRE code}

As mentioned above, the code STAPRE uses the HauserFeshbach formalism for equilibrium processes and the exciton model for pre-equilibrium (PE) emission. For the latter, this concerns the exciton model with one component in contrast with the TALYS calculations. The transmission coefficients for neutrons, protons, deuterons and alpha particles were provided as input data to the STAPRE code by means of the spherical optical model code SCAT-2 [55] using global parameter sets. The neutron optical model parameters were tested by comparing the experimental total cross section data with results of the model calculation. The best description was given by the Bersillon-Cindro potential [38]. Above $1 \mathrm{MeV}$ neutron energy, the calculation shows quite good agreement with the data. Below $1 \mathrm{MeV}$ neutron energy the deviation between the measured and calculated cross section increases for all tested potentials. The calculated data using the potential of Koning-Delaroche [43] show similar behavior but the data are lower than those given by the Bersillon-Cindro potential. For protons, the optical model parameter set of Ref. [56] was used. In the case of alpha particles, the parameters proposed in Ref. [57] were modified to obtain a better description of the data. For the energy and mass dependence of the effective matrix element in the exciton model, $|M|^{2}=(\mathrm{FM}) A^{-3} E^{-1}$ was applied. The energies, spins, parities, and branching ratios of the discrete levels were obtained from the evaluated nuclear structure and decay database ENSDF [51]. In cases where the spin and parity were not known, estimates from adjacent levels were made. In the continuum region two types of level density formulas were used for which very comparable results were obtained:

(i) The back-shifted Fermi-gas (BSFG) formula with the level density parameter given in Refs. [58,59]. The level density parameter $a$ for the calculation was selected by interpolating the data of the neighboring isotopes, taking into account the odd-even systematics. The back-shift parameter $(\Delta)$ was determined individually for all nuclei
TABLE III. Level density parameters used in the STAPRE model calculation. As a starting point the RIPL-2 [38] database was used, but some parameters were adjusted to match the data.

\begin{tabular}{|c|c|c|c|c|c|}
\hline \multirow[t]{2}{*}{ Isotope } & \multicolumn{2}{|c|}{$\eta=1$} & \multicolumn{2}{|c|}{$\eta=0.5$} & \multirow{2}{*}{$\frac{E_{d \max }}{(\mathrm{MeV})}$} \\
\hline & $a$ & $\Delta$ & $a$ & $\Delta$ & \\
\hline${ }^{202} \mathrm{Hg}$ & 14.85 & 0.1 & 13.53 & 0.1 & 2 \\
\hline${ }^{203} \mathrm{Hg}$ & 13.06 & -0.85 & 11.5 & -1.05 & 1 \\
\hline${ }^{204} \mathrm{Hg}$ & 14.38 & 0.6 & 12.85 & 0.5 & 1.4 \\
\hline${ }^{200} \mathrm{Tl}$ & 15.6 & -0.55 & 14.08 & -0.5 & 0.7 \\
\hline${ }^{201} \mathrm{Tl}$ & 13.04 & -0.4 & 11.67 & -0.4 & 2 \\
\hline${ }^{202} \mathrm{Tl}$ & 14.21 & -0.55 & 13 & -0.6 & 0.9 \\
\hline${ }^{203} \mathrm{Tl}$ & 13.06 & -0.5 & 11.77 & -0.6 & 1.8 \\
\hline${ }^{207} \mathrm{Tl}$ & 11.79 & 1.1 & 10.28 & 0.95 & 2.5 \\
\hline${ }^{208} \mathrm{Tl}$ & 11.24 & -0.89 & 9.61 & -1.06 & 1 \\
\hline${ }^{201} \mathrm{~Pb}$ & 13.04 & -0.5 & 11.67 & -0.5 & 1.2 \\
\hline${ }^{202} \mathrm{~Pb}$ & 14.85 & 0.65 & 13.53 & 0.6 & 2.2 \\
\hline${ }^{203} \mathrm{~Pb}$ & 13.06 & -0.65 & 11.65 & -0.8 & 1.2 \\
\hline${ }^{204} \mathrm{~Pb}$ & 14.38 & 0.3 & 12.68 & -0.2 & 2 \\
\hline${ }^{205} \mathrm{~Pb}$ & 13.97 & -0.65 & 12.6 & -0.7 & 1 \\
\hline${ }^{206} \mathrm{~Pb}$ & 13.28 & 0.25 & 12.00 & 0.15 & 1.5 \\
\hline${ }^{207} \mathrm{~Pb}$ & 11.79 & 0.95 & 10.35 & 0.86 & 2.7 \\
\hline${ }^{208} \mathrm{~Pb}$ & 11.38 & 2.07 & 9.7 & 1.8 & 5 \\
\hline${ }^{209} \mathrm{~Pb}$ & 10.31 & 0.23 & 8.47 & -0.3 & 3 \\
\hline
\end{tabular}

used in the model calculation. The cumulative plot of the known discrete levels, collected from the ENSDF database, was fitted by the BSFG formula while the level spacing at the neutron binding energy was kept according to the experimental value. The values are shown in Table III.

(ii) An energy dependent level density parameter $(a)$ :

$$
a(U, Z, A)=\tilde{a}(A)\left(1+\frac{\delta E_{0}}{U}[1-\exp (-\gamma U)]\right) .
$$

In the case of the energy dependent level-density parameters, the data of the RIPL-2 [38] file of Beijing were used for the isotopes available in that file. For nuclei without data in the RIPL file, the asymptotic level density parameter $\tilde{a}(A)=0.073 A+0.115 A^{2 / 3} \mathrm{MeV}^{-1}$ was used following the RIPL recommendation. The other parameters were fixed so that the energy dependent level-density parameter would equal the BSFG parameter at the binding energy of the neutron and so that the cumulative number of discrete levels is matched.

The spin distribution of the level density was characterized by the ratio $\eta$ of the effective moment of inertia $\Theta_{\text {eff }}$ to the rigid-body moment of inertia $\Theta_{\text {rig }}\left(\eta=\Theta_{\text {eff }} / \Theta_{\text {rig }}, \Theta_{\text {rig }}=\right.$ $1.25 \mathrm{~A}^{1 / 3}$ ) and the calculations were performed for $\eta=1.0$ and 0.5 . The transmission coefficients of photons are also of considerable significance in calculations of isomeric cross sections. They were derived from the gamma-ray strength functions. For the $E 1$ transition the Brink-Axel model with global parameters was applied, while for the $M 1, E 2, M 2, E 3$, and $M 3$ radiation the Weisskopf model was used. 
TABLE IV. Measured reaction cross sections for the charged particle emission reactions. Standard uncertainties are given for the cross sections. The standard spreads are given for the neutron energy distributions.

\begin{tabular}{lccc}
\hline \hline \multicolumn{4}{c}{${ }^{206} \mathrm{~Pb}(n, \alpha)^{203} \mathrm{Hg}$} \\
\hline$E_{n}(\mathrm{MeV})$ & $\sigma(\mathrm{mb})$ & $E_{n}(\mathrm{MeV})$ & $\sigma(\mathrm{mb})$ \\
\hline $16.1 \pm 0.2$ & $1.12 \pm 0.13$ & $17.2 \pm 0.1$ & $1.9 \pm 0.2$ \\
$18.7 \pm 0.2$ & $2.6 \pm 0.2$ & $19.2 \pm 0.2$ & $3.3 \pm 0.3$ \\
$20.0 \pm 0.1$ & $3.9 \pm 0.4$ & $20.5 \pm 0.1$ & $3.7 \pm 0.4$ \\
\multicolumn{4}{c}{${ }^{208} \mathrm{~Pb}(n, p)^{208} \mathrm{Tl}$} \\
$14.81 \pm 0.17$ & $0.95 \pm 0.22$ & $16.3 \pm 0.2$ & $2.1 \pm 0.3$ \\
$17.95 \pm 0.12$ & $4.2 \pm 0.4$ & $19.05 \pm 0.09$ & $6.1 \pm 0.4$ \\
$19.3 \pm 0.09$ & $6.1 \pm 0.5$ & $20.5 \pm 0.08$ & $8.1 \pm 0.5$ \\
\hline \hline
\end{tabular}

\section{RESULTS AND DISCUSSION}

The results of the experiments are presented in Tables IV and V. In Figs. 1 and 2 they are compared with earlier measurements and the model calculations. The uncertainty of $E_{n}$ given in the tables and graphs is the standard spread in the neutron energies with which the samples were irradiated [7]. The uncertainties [11] of the measured cross sections were obtained combining the individual uncertainties in quadrature. Reactions involving charged particle emission are presented in Sec. IV A, neutron emission reactions in Sec. IV B.

TABLE V. Measured reaction cross sections for the neutron emission reactions. Standard uncertainties are given for the cross sections. The standard spreads are given for the neutron energy distributions.

\begin{tabular}{|c|c|c|c|}
\hline \multicolumn{4}{|c|}{${ }^{204} \mathrm{~Pb}\left(n, n^{\prime} \gamma\right){ }^{204} \mathrm{~Pb}^{m}$} \\
\hline$E_{n}(\mathrm{MeV})$ & $\sigma(\mathrm{mb})$ & $E_{n}(\mathrm{MeV})$ & $\sigma(\mathrm{mb})$ \\
\hline $3.45 \pm 0.03$ & $43 \pm 3$ & $14.81 \pm 0.17$ & $62 \pm 4$ \\
\hline $16.26 \pm 0.20$ & $51 \pm 3$ & $17.95 \pm 0.12$ & $42 \pm 5$ \\
\hline $19.39 \pm 0.09$ & $36 \pm 3$ & $20.53 \pm 0.08$ & $27 \pm 5$ \\
\hline \multicolumn{4}{|c|}{${ }^{204} \mathrm{~Pb}(n, 2 n){ }^{203} \mathrm{~Pb}$} \\
\hline $14.81 \pm 0.17$ & $2146 \pm 133$ & $15.55 \pm 0.25$ & $2163 \pm 109$ \\
\hline $16.26 \pm 0.20$ & $2154 \pm 114$ & $18.0 \pm 0.1$ & $2007 \pm 185$ \\
\hline $18.7 \pm 0.2$ & $1923 \pm 147$ & $19.2 \pm 0.2$ & $1852 \pm 132$ \\
\hline $19.4 \pm 0.1$ & $1827 \pm 138$ & $20.5 \pm 0.1$ & $1461 \pm 140$ \\
\hline $20.5 \pm 0.1$ & $1544 \pm 108$ & & \\
\hline \multicolumn{4}{|c|}{${ }^{204} \mathrm{~Pb}(n, 2 n)^{203} \mathrm{~Pb}^{m 1}$} \\
\hline $16.26 \pm 0.20$ & $1039 \pm 72$ & $17.95 \pm 0.12$ & $1030 \pm 70$ \\
\hline $19.11 \pm 0.09$ & $1032 \pm 82$ & $20.31 \pm 0.08$ & $828 \pm 73$ \\
\hline \multicolumn{4}{|c|}{${ }^{204} \mathrm{~Pb}(n, 3 n)^{202} \mathrm{~Pb}^{m}$} \\
\hline $18.0 \pm 0.1$ & $0.17 \pm 0.07$ & $18.7 \pm 0.2$ & $3.8 \pm 0.5$ \\
\hline $19.2 \pm 0.2$ & $13.4 \pm 2.2$ & $19.4 \pm 0.1$ & $18.3 \pm 2.2$ \\
\hline $20.5 \pm 0.1$ & $82 \pm 14$ & & \\
\hline \multicolumn{4}{|c|}{${ }^{206} \mathrm{~Pb}(n, 3 n)^{204} \mathrm{~Pb}^{m}$} \\
\hline $18.1 \pm 0.1$ & $4.2 \pm 0.3$ & $19.4 \pm 0.1$ & $92.1 \pm 6.4$ \\
\hline $20.7 \pm 0.1$ & $292 \pm 31$ & & \\
\hline
\end{tabular}

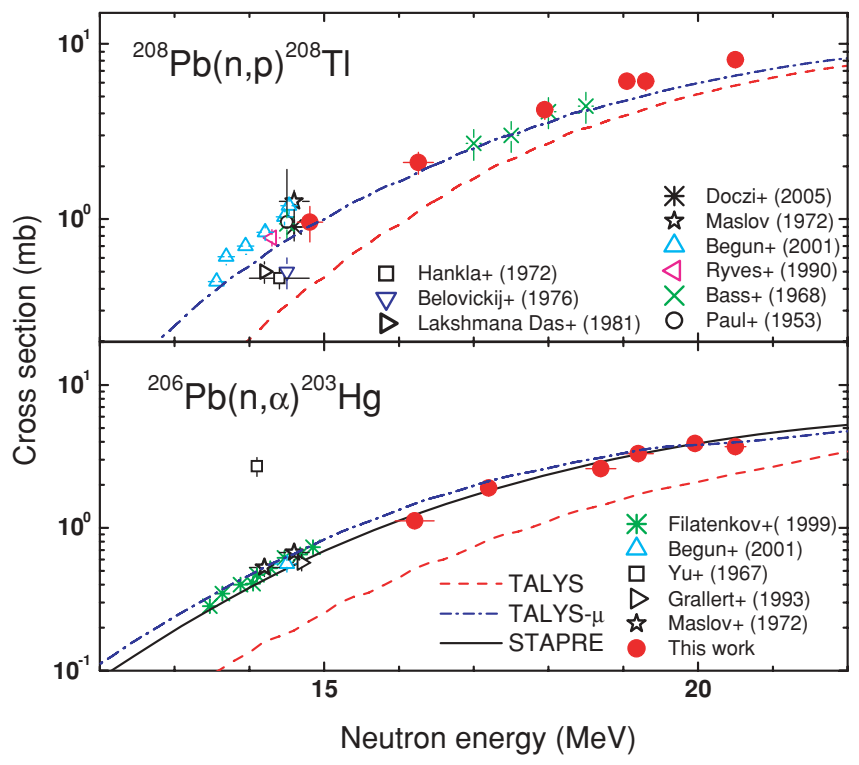

FIG. 1. (Color online) Experimental results for the charged particle emission reactions compared with results from earlier measurements and the TALYS and STAPRE model calculations.

\section{A. Charged particle emission}

Two reactions were studied for which charged particles were emitted, the ${ }^{208} \mathrm{~Pb}(n, p)^{208} \mathrm{Tl}$ reaction and the ${ }^{206} \mathrm{~Pb}(n, \alpha){ }^{203} \mathrm{Hg}$ reaction.

\section{The ${ }^{208} \mathrm{~Pb}(n, p){ }^{208} \mathrm{Tl}$ reaction}

The study of this reaction on natural lead is free from interfering reactions. A pneumatic transport system was used to achieve sufficient statistics under conditions that were optimised for the study of the ${ }^{204} \mathrm{~Pb}(n, 2 n)^{203} \mathrm{~Pb}^{m 1}$ reaction. Control measurements at two energies validated the procedure, as described in Sec. II. Despite the low threshold of this reaction, corrections for low energy neutrons are small since the cross section falls very rapidly for energies less than $10 \mathrm{MeV}$ due to the Coulomb barrier.

The present results consist of six points from 14.8 to $20.5 \mathrm{MeV}$. At $14.8 \mathrm{MeV}$ the current work agrees well with the results of Refs. [60-64], and not with those of Refs. [65-67]. The new data agree with the results of Bass and Wechsung [68], in the energy range from 17 to $18.5 \mathrm{MeV}$. Not shown, is a clear discrepancy with the results of Ref. [69] that are 3 to 5 times higher than the present data between 20 and $22 \mathrm{MeV}$.

The TALYS- $\mu$ calculation is in good agreement with the data at low energy while being somewhat below the experimental results above $18 \mathrm{MeV}$. In contrast the TALYS calculation is significantly below the data in the entire range. To understand the differences between the two TALYS calculations a number of additional calculations were made to try to identify their causes. First, switching off pre-equilibrium emission reveals that for the TALYS- $\mu$ calculation evaporated protons dominate below $15 \mathrm{MeV}$ and are rapidly negligible above, while for the TALYS calculation these are negligible throughout. Second, the phenomenological optical model in the TALYS calculation was replaced with that of the TALYS $\mu$ calculation. At $20 \mathrm{MeV}$ this 


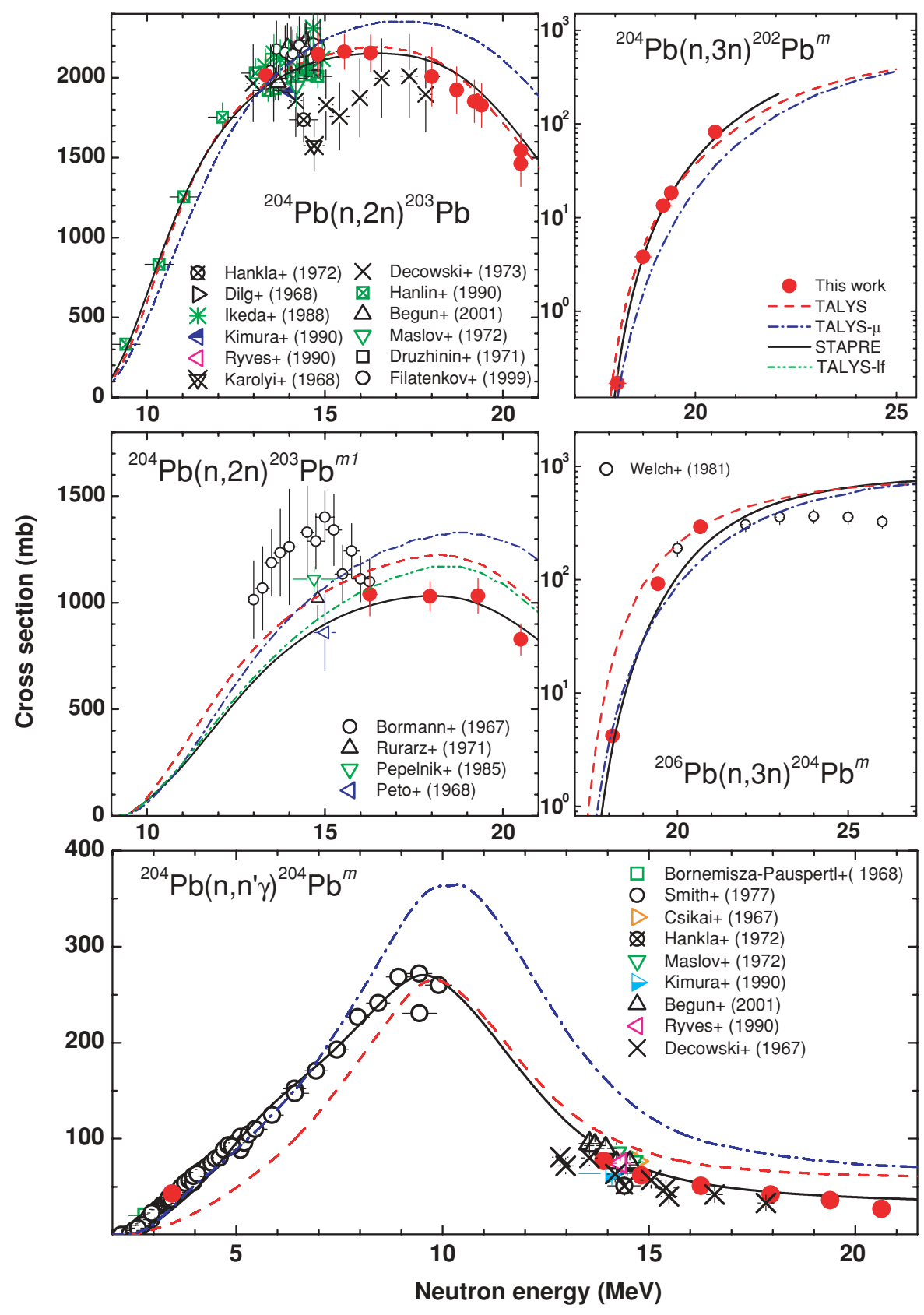

FIG. 2. (Color online) Experimental results for the neutron emission reactions compared with results from earlier measurements and the TALYS and STAPRE model calculations. explains the difference nearly completely while near $15 \mathrm{MeV}$ this only covers for about $1 / 3$ of the difference (on a logarithmic scale). Clearly, the level density models differ such that in one case evaporation plays a role and the other not at all. We note that for lead isotopes, sensitivity to the level density may be stronger than for any other stable nuclide in the periodic table. It is well known that here the shell effects are very strong at low energies, and also that the shell effects are damped quickly with increasing energy. This means that different level density models, phenomenological or microscopic, may well describe the discrete levels and the mean resonance spacing but may behave very differently in energy regions (e.g., between 3 and $8 \mathrm{MeV}$ ) where such experimental probes are not available. Such large differences may give rise to significant differences in cross section predictions.
The difference between TALYS and TALYS- $\mu$ at energies above $15 \mathrm{MeV}$ is due to the proton optical model for ${ }^{208} \mathrm{Tl}$ that determines the pre-equilibrium emission rates since these are proportional to the cross section for the inverse process (microreversibility/detailed balance).

\section{The ${ }^{206} \mathrm{~Pb}(n, \alpha){ }^{203} \mathrm{Hg}$ reaction}

To determine the activity $\gamma$-ray spectrometry was performed following a cooling time sufficient ( $>1$ month) to guarantee negligible $(<1 \%)$ contributions from ${ }^{203 g} \mathrm{~Pb}$, which emits the same $\gamma$-ray $(279 \mathrm{keV})$. The latter activity is produced by the ${ }^{204} \mathrm{~Pb}(n, 2 n){ }^{203} \mathrm{~Pb}$ reaction (see below). The six experimental results of this work are the first in the energy range from 16 to $20.5 \mathrm{MeV}$. The excitation function formed by the present 
results and those at $14 \mathrm{MeV}[61,63,70,71]$ is in good agreement with the results from the TALYS- $\mu$ and the STAPRE calculations. The point of Ref. [72] is clearly out. Agreement with the TALYS$\mu$ calculation, which is entirely based on systematics and the folding approach of Watanabe to the optical model, seems remarkable in view of the common difficulty of reproducing reactions in which complex particles are emitted. On the other hand the phenomenological TALYS calculation based on global systematics is clearly below the data. It was verified with a similar check as described in the previous section, that there is no difference with the TALYS- $\mu$ calculation on account of a difference in the underlying nucleon-optical model. So, here the differences are primarily due to a difference in the level densities of the TALYS and TALYS- $\mu$ calculations. Switching off pre-equilibrium emission revealed that evaporation of alpha-particles is negligible for the TALYS calculation, while it dominates for the TALYS- $\mu$ calculation up to $18 \mathrm{MeV}$, providing still $40 \%$ near $20 \mathrm{MeV}$. The calculations with the STAPRE code are in excellent agreement with the data, primarily on account of the alpha-particle optical model.

\section{B. Neutron emission cross sections}

Measured neutron emission cross sections consist of one full $(n, 2 n)$ cross section of ${ }^{204} \mathrm{~Pb}$ and four cross sections leading to an isomer. Four of the reactions in this section have ${ }^{204} \mathrm{~Pb}$ as target nucleus.

\section{1. $T h e^{204} \mathrm{~Pb}\left(n, n^{\prime} \gamma\right){ }^{204} \mathrm{~Pb}^{m}$ reaction}

The study of this reaction was greatly facilitated by the use of enriched sample material. The latter was even essential to account for interference above the threshold of the ${ }^{206} \mathrm{~Pb}(n, 3 n)^{204} \mathrm{~Pb}^{m}$ reaction discussed below. Five experimental results were obtained from 13 to $21 \mathrm{MeV}$ and one at $3.45 \mathrm{MeV}$. The two highest energy results were corrected for the contribution of the ${ }^{206} \mathrm{~Pb}(n, 3 n)^{204} \mathrm{~Pb}^{m}$ reaction. An extensive set of measurements was already carried out by Smith and Meadows [73] in the range from 2.3 to $10 \mathrm{MeV}$ using a highly enriched sample of ${ }^{204} \mathrm{~Pb}$. The present result at 3.45 MeV incident neutron energy is in excellent agreement with that work, as is the result of [74] for $2.8 \mathrm{MeV}$. At higher energies the present work is in good agreement with most of the results obtained at $14 \mathrm{MeV}$ [61-63,65,75,76]. Above $14 \mathrm{MeV}$ the only other result is by Decowski et al. [77]. The latter work agrees rather well with the present results but appears to be systematically somewhat low with regard to most of the data. The STAPRE calculations reproduce the data of this work very well irrespective of the various options that were tested. However, through comparison with the data at lower energy it appeared that near the maximum the $\eta=1$ results were significantly too high. Less significantly, the energy dependent level density was somewhat favored over the constant level density parameters. Neither the TALYS nor the TALYS- $\mu$ calculations show quantitative agreement with the experimental results of this work with the exception of the result at $3.45 \mathrm{MeV}$ that is on top of the TALYS- $\mu$ prediction. The latter agrees well with the low energy data but is generally too high above $8 \mathrm{MeV}$. The maximum of the TALYS calculation is reasonably close to the maximum of the data in energy and cross section but the shape of the predicted cross section is at variance with that of the data. It must be acknowledged that here the main difference in the TALYS and TALYS $-\mu$ calculations lie in the level densities while for this cross section the pre-equilibrium contribution dominates above $13 \mathrm{MeV}$. The sensitivity to level densities is therefore small in this energy range. Thus, also the difference between the two TALYS calculations that have the same pre-equilibrium contribution is indicative of a difference in absorption cross sections.

\section{The ${ }^{204} \mathrm{~Pb}(n, 2 n)^{203} \mathrm{~Pb}$ reaction}

The experimental results for the ${ }^{204} \mathrm{~Pb}(n, 2 n){ }^{203} \mathrm{~Pb}$ reaction were obtained using about $4.5 \mathrm{~g}$ of natural lead. Activity determinations were performed within several hours following irradiation but allowing several minutes for the decay of the short-lived isomers. Thus, the cross sections obtained are free from interference $(<1 \%)$ of the ${ }^{206} \mathrm{~Pb}(n, \alpha){ }^{203} \mathrm{Hg}$ reaction and constitute the total $(n, 2 n)$ cross section, i.e., not that of the prompt production of ${ }^{203 g} \mathrm{~Pb}$. Gamma-ray attenuation corrections were reduced by spreading the foils of the irradiated stack over the detector.

Nine experimental results were obtained in the neutronenergy range from 14.8 to $20.5 \mathrm{MeV}$ with overall uncertainties between 5 and $10 \%$. Above $18 \mathrm{MeV}$ the results are unique and above $16 \mathrm{MeV}$ the only other data are those of Decowski et al. [77] which have a much larger uncertainty and a peculiar shape around $14 \mathrm{MeV}$. Together with the results of Hanlin et al. [78] and the average of the results around $14 \mathrm{MeV}$ [61-63,65,70,76,79-82] our data establish an excitation curve for this reaction from threshold to $20 \mathrm{MeV}$.

The experimentally determined excitation curve is in excellent agreement with the model calculations based on phenomenology. Both the globally optimized parameters used for the TALYS calculation and that for the optimized set of STAPRE perform very well. For STAPRE the results with $\eta=0.5$ are shown and level density option a. Similar results are obtained with the other options (see Sec. III). In contrast, the TALYS- $\mu$ calculation is progressively above the experimental results from this work, agreeing well only from 15 to $16.5 \mathrm{MeV}$. At lower energies it falls below the experimental results of Ref. [78]. Since this channel constitutes a large fraction of the nonelastic cross section in the energy range, good(poor) agreement essentially corroborates(contests) the neutron optical model. In addition, near the rise and fall of the cross section, level-density driven competition with other main channels plays an important role. To some extent preequilibrium emission depletes the $(n, 2 n)$ channel and enhances inelastic scattering. However, again the main sensitivity is due the optical model through the proportionality of the pre-equilibrium cross section to the cross section of the inverse process. It was found that the sensitivity to parameters governing the pre-equilibrium process is significantly less than to optical model parameters controlling the absorption cross section. 


\section{The ${ }^{204} \mathrm{~Pb}(n, 2 n){ }^{203} \mathrm{~Pb}^{m 1}$ reaction}

As described in Sec. II, this reaction was studied using an automated radiation-counting sequence to obtain sufficient statistics for the determination of the activity of the short-lived isomer ${ }^{203} \mathrm{~Pb}^{m}\left(T_{1 / 2}=6.21 \mathrm{~s}\right)$. Theoretically, the results of this measurement could be influenced by a second isomer $\left(E_{x}=2948 \mathrm{keV}, T_{1 / 2}=0.48 \mathrm{~s}, J^{\pi}=29 / 2^{-}\right)$. As the transfer time between each irradiation and counting period is $4 \mathrm{~s}$, only $0.3 \%$ of the initial population of the $0.48 \mathrm{~s}$ isomer does not contribute directly to the activity of the $6.21 \mathrm{~s}$ isomer. On top of that, in view of the spin and excitation energy of the $0.48 \mathrm{~s}$ isomer, its population in a neutron-induced reaction with incident energies below $21 \mathrm{MeV}$ will be less than that for the $6.21 \mathrm{~s}$ isomer. Therefore, the present results represent the cross section that corresponds to the full decay of the $0.48 \mathrm{~s}$ isomer.

Four experimental results were obtained from 16.3 to 20.3 MeV of which three are unique in the energy range studied. At the lowest energy there is agreement with the highest energy result by Bormann et al. [83]. However, in general, the status of the data between 12 and $16 \mathrm{MeV}$ is confusing [83-86]. The new results are well reproduced by the STAPRE model calculations that also agree well with the experimental work of Ref. [84]. Here, the result for $\eta=0.5$ is shown with level density option (i) of the previous section. Level density option (ii) gives similar results, while the calculation with $\eta=1$ is clearly above the data (10-20\%, not shown). The TALYS calculation agrees reasonably well in shape but is $10-20 \%$ above the data. The TALYS- $\mu$ calculation depicts the same behavior relative to the TALYS calculation as shown for the previous reaction, and accordingly agrees less with the data.

The modeling of isomer cross sections is more delicate since it requires good accounting of excitation and decay of levels in the final nucleus. On the one hand this is demonstrated by the sensitivity to $\eta$, the parameter that controls the spin dependence of the level density. Here, this is also partially illustrated with the TALYS-lf calculation. In this calculation we have included a level and decay scheme that is based on the most recent ENSDF evaluation [24] for $A=203$. In contrast, the TALYS, TALYS- $\mu$, and STAPRE calculations use the previous evaluated data set which differs in the first 25 levels and their decay. As a result the cross section below $20 \mathrm{MeV}$ is reduced compared to that of the TALYS calculation resulting in an improved agreement with the data. In this work, this is the only isomer cross section for which level and decay data play a role. The others are exclusively determined by feeding from states in the continuum.

\section{4. $T h e^{204} \mathrm{~Pb}(n, 3 n)^{202} \mathrm{~Pb}^{m}$ reaction}

The present measurements resulted in the first five data points reported for the ${ }^{204} \mathrm{~Pb}(n, 3 n)^{202} \mathrm{~Pb}^{m}$ reaction. Unambiguous results were obtained since experimentally this channel is free from interferences. The data represent part of the rising edge of the excitation function. The TALYS and STAPRE calculations both show excellent agreement with the measurements. In contrast the TALYS- $\mu$ predictions are significantly below the experimental results. To pinpoint the cause of this discrepancy is more complicated since an isomer cross section is only a fraction of the $(n, 3 n)$ channel. However, the results shown above indicate that TALYS- $\mu$ gives too small a cross section for the ${ }^{204} \mathrm{~Pb}(n, 3 n){ }^{202} \mathrm{~Pb}$ reaction as a result of a too high cross section for the ${ }^{204} \mathrm{~Pb}(n, 2 n)^{203} \mathrm{~Pb}$ reaction. This would indicate that the level density model should be adjusted to improve agreement with the data. This suggestion was verified replacing in the TALYS calculation the phenomenological optical model with the microscopic optical model. No appreciable difference with the TALYS calculation was observed.

For STAPRE only the result with $\eta=0.5$ is shown. For $\eta=$ 1 , results are clearly above the data for $E_{n}>19 \mathrm{MeV}$. The difference amounts to $20 \%$ at $20.5 \mathrm{MeV}$. The two options for the level density tested with this code perform equally well.

\section{The ${ }^{206} \mathrm{~Pb}(n, 3 n)^{204} \mathrm{~Pb}^{m}$ reaction}

The other $(n, 3 n)$ process investigated is the ${ }^{206} \mathrm{~Pb}(n$, $3 n)^{204} \mathrm{~Pb}^{m}$ reaction, for which three points were obtained using a highly enriched ${ }^{206} \mathrm{~Pb}$ sample. This sample was free of ${ }^{204} \mathrm{~Pb}$ so that no consideration had to be given to the inelastic excitation of the isomer. The only previous experimental work by Welch et al. [69] covers the range from 18 to $26 \mathrm{MeV}$. The result at the lowest energy agrees with the present work, but the trend at higher energies does not appear to be a natural extension of the trend suggested by this work. The three model calculations do not provide any quantitative agreement with the data. The TALYS calculation agrees well with the present work for 19.4 and $20.7 \mathrm{MeV}$. It is however significantly above the $18.1 \mathrm{MeV}$ experimental result. The TALYS- $\mu$ and STAPRE results agree with the data at $18.1 \mathrm{MeV}$ but are below the results at 19.4 and $20.7 \mathrm{MeV}$. All three calculations do not follow the trend suggested by the data of Welch et al. and predict higher maximum cross sections that occur at a higher energy than follows from the data.

\section{SUMMARY}

New experimental results were obtained for the ${ }^{204} \mathrm{~Pb}\left(n, n^{\prime} \gamma\right){ }^{204} \mathrm{~Pb}^{m},{ }^{204} \mathrm{~Pb}(n, 2 n){ }^{203} \mathrm{~Pb},{ }^{204} \mathrm{~Pb}(n, 2 n){ }^{203} \mathrm{~Pb}^{m 1}$, $\left.{ }^{204} \mathrm{~Pb}(n, 3 n)\right)^{202} \mathrm{~Pb}^{m},{ }^{206} \mathrm{~Pb}(n, 3 n){ }^{204} \mathrm{~Pb}^{m},{ }^{206} \mathrm{~Pb}(n, \alpha){ }^{203} \mathrm{Hg}$, and ${ }^{208} \mathrm{~Pb}(n, p){ }^{208} \mathrm{Tl}$ reaction cross sections at the IRMM van de Graaff laboratory in the neutron energy range from 14 to $21 \mathrm{MeV}$. Natural and enriched samples were irradiated with neutrons produced via the ${ }^{3} \mathrm{H}(d, n)^{4} \mathrm{He}$ reaction. The induced activities were determined by gamma-ray spectrometry using HPGe detectors in a low-background shields. Neutron fluences were determined with the well-known cross section of the ${ }^{27} \mathrm{Al}(n, \alpha){ }^{24} \mathrm{Na}$ reaction. Contributions from spurious low energy neutrons were estimated with five additional activation standards and experimental knowledge about the neutron spectrum from time-of-flight measurements. Enriched samples were essential to determine the cross sections for the reactions with ${ }^{204} \mathrm{~Pb}^{m}$ and ${ }^{206} \mathrm{~Pb}^{m}$ isomers in the final state. In this energy range these can be excited by inelastic scattering and by $(n, 2 n)$ and $(n, 3 n)$ reactions on the stable heavier isotopes. Accurate 
results for reactions with ${ }^{204,206} \mathrm{~Pb}$ as target nuclei with natural lead samples were enabled through a precise measurement of the isotopic ratios.

For a first investigation of the consequences of the present data for nuclear reaction models they were confronted with three calculations. Two of these, using on the one hand the default phenomenological approach and on the other hand the optional microscopic approach of the TALYS code, involved global parameter systematics and avoided any matching to the present data. For the third calculation with the phenomenological approach of the STAPRE code, parameters were carefully adjusted to reproduce the available data for lead, including those of the present work.

The results of the TALYS phenomenological approach show excellent agreement with the present data for the ${ }^{204} \mathrm{~Pb}(n, 2 n){ }^{203} \mathrm{~Pb}$ and ${ }^{204} \mathrm{~Pb}(n, 3 n)^{202} \mathrm{~Pb}^{m}$ reactions, reasonable agreement with the ${ }^{204} \mathrm{~Pb}(n, 2 n)^{203} \mathrm{~Pb}^{m 1}$ and ${ }^{206} \mathrm{~Pb}(n, 3 n)^{204} \mathrm{~Pb}^{m}$ reactions, but differ significantly for the ${ }^{204} \mathrm{~Pb}\left(n, n^{\prime} \gamma\right){ }^{204} \mathrm{~Pb}^{m},{ }^{206} \mathrm{~Pb}(n, \alpha){ }^{203} \mathrm{Hg}$ and ${ }^{208} \mathrm{~Pb}(n, p){ }^{208} \mathrm{Tl} \mathrm{re}-$ actions. For the TALYS microscopic approach results are excellent for the $\left.{ }^{206} \mathrm{~Pb}(n, \alpha)\right)^{203} \mathrm{Hg}$ and ${ }^{208} \mathrm{~Pb}(n, p){ }^{208} \mathrm{Tl}$ reactions but differ significantly with the measured data for the other reactions. The discrepancy with the ${ }^{204} \mathrm{~Pb}(n, 2 n)^{203} \mathrm{~Pb}$ reaction cross section is perhaps most surprising, as this reaction exhausts a large fraction of the reaction cross section and a good prediction would be expected. In this context, it may be noted that the microscopic approach compared favourably with the phenomenological approach for the measured ${ }^{208} \mathrm{~Pb}(n, x n \gamma)$ cross sections of Ref. [3]. Although the present results for ${ }^{208} \mathrm{~Pb}$ show that this nucleus is modeled well also for the $(n, p)$ channel, a good quantitative agreement is apparently not uniformly extended to reactions on ${ }^{204,206} \mathrm{~Pb}$. A positive surprise is the good agreement for the ${ }^{206} \mathrm{~Pb}(n, \alpha){ }^{203} \mathrm{Hg}$ reaction. Generally good agreement is more difficult to obtain for reactions involving the emission of complex particles. The calculations using the STAPRE code with carefully selected parameters agree well with the data in all cases, except for the ${ }^{204} \mathrm{~Pb}(n, 3 n){ }^{202} \mathrm{~Pb}^{m}$ reaction. It was verified that the measured cross sections for reactions leading to isomers in the final state are more readily reproduced using a moment of inertia that is half rather than the full moment of inertia of a rigid rotator. This is in line with earlier findings for reactions leading to isomers of $\mathrm{Au}$ and $\mathrm{Hg}$ [4,5].

In summary the present work contributes valuable new data for neutron-induced reactions on isotopes of lead and in particular on ${ }^{204} \mathrm{~Pb}$. It was shown that agreement with globally optimized nuclear model calculations varies on a case by case basis. It is therefore expected that the present findings will contribute to further improvements in the modeling of neutron-induced nuclear reactions and in particular with regard to the production of isomers.

\section{ACKNOWLEDGMENTS}

The authors thank the Van de Graaff operators for the irradiation conditions that made this work possible and A. Moens for the preparation of the samples. P.R. and V.S. are grateful to the Commission of the European Communities for financial support.
[1] C. Mahaux and R. Sartor, Nucl. Phys. A516, 285 (1990).

[2] L. C. Mihailescu, C. Borcea, A. Koning, A. Pavlik, and A. J. M. Plompen, Nucl. Phys. A799, 1 (2008).

[3] L. C. Mihailescu, C. Borcea, P. Baumann, P. Dessagne, E. Jericha, H. Karam, M. Kerveno, A. J. Koning, N. Leveque, A. Pavlik et al., Nucl. Phys. A811, 1 (2008).

[4] M. Al-Abyad, S. Sudár, M. N. H. Comsan, and S. M. Qaim, Phys. Rev. C 73, 064608 (2006).

[5] S. Sudár and S. M. Qaim, Phys. Rev. C 73, 034613 (2006).

[6] D. Rochman and A. J. Koning, Nucl. Instrum. Methods Phys. Res. A 589, 85 (2008).

[7] P. Reimer, V. Avrigeanu, S. V. Chuvaev, A. A. Filatenkov, T. Glodariu, A. Koning, A. J. M. Plompen, S. M. Qaim, D. L. Smith, and H. Weigmann, Phys. Rev. C 71, 044617 (2005).

[8] V. Semkova, V. Avrigeanu, T. Glodariu, A. J. Koning, A. J. M. Plompen, D. L. Smith, and S. Sudár, Nucl. Phys. A730, 255 (2004).

[9] P. Reimer, M. Hult, A. J. M. Plompen, P. N. Johnston, V. Avrigeanu, and S. M. Qaim, Nucl. Phys. A705, 265 (2002).

[10] P. Reimer, V. Avrigeanu, A. J. M. Plompen, and S. M. Qaim, Phys. Rev. C 65, 014604 (2001).

[11] A. Fessler, A. J. M. Plompen, D. L. Smith, J. W. Meadows, and Y. Ikeda, Nucl. Sci. Eng. 134, 171 (2000).

[12] V. Semkova, V. Avrigeanu, A. J. M. Plompen, P. Reimer, D. L. Smith, S. Sudár, A. Koning, and A. Forrest, Neutron activation cross sections for safety of nuclear power plants, EUR Report
20820 EN, ISBN 92-894-6095-4, European Communities, JRCIRMM, Geel, Belgium (2003).

[13] A. Fessler and S. M. Qaim, Radiochimica acta 84, 1 (1999).

[14] K. J. R. Rosman and P. D. P. Taylor, Pure Appl. Chem. 70, 217 (1998).

[15] R. D. Russell and R. M. Farquar, Lead Isotopes In Geology (Interscience Publisher Inc., New York, 1960).

[16] G. Lövestam, EnergySet, EC-JRC-IRMM (private communication, 2004).

[17] M. Drosg, DROSG-2000: Neutron Source Reactions, Report IAEA-NDS-87, v. 2.2, Rev. 8, IAEA, Vienna, Austria, wwwnds.iaea.org (2003).

[18] J. F. Ziegler, SRIM 2003: The Stopping and Range of Ions in Matter, www.srim.org (2003).

[19] D. Schlegel, TARGET User's manual, Laborbericht PTB-6.42-052, Braunschweig (2005).

[20] National Nuclear Data Center, information extracted from the NuDat database, http://www.nndc.bnl.gov/nudat2 (2008).

[21] R. Macfarlane, Qtool: Calculation of reaction Q-values and thresholds, T-2 Nuclear Information Service, Los Alamos National Laboratory, USA, March 1997, t2.lanl.gov/data/qtool. html (1997).

[22] G. Audi, A. H. Wapstra, C. Thibault, J. Blachot, and O. Bersillon, Nucl. Phys. A729, 337 (2003).

[23] S. Zhu and F. G. Kondev, Nucl. Data Sheets 109, 699 (2008).

[24] F. G. Kondev, Nucl. Data Sheets 105, 1 (2005).

[25] M. R. Schmorak, Nucl. Data Sheets 72, 409 (1994). 
[26] M. J. Martin, Nucl. Data Sheets 108, 1583 (2007).

[27] A. B. Smith, S. Chiba, D. L. Smith, J. W. Meadows, P. T. Guenther, R. D. Lawson, and R. J. Howerton, Evaluated Neutronic File for Indium, Argonne National Laboratory, Report No. ANL/NDM-115 (1990).

[28] V. McLane, Evaluated Nuclear Data File, Section B, Version VI (ENDF/B-VI), National Nuclear Data Center, Brookhaven National Laboratory (1998), available online at www.nndc.bnl.gov/nndc/endf.

[29] M. Wagner, H. Vonach, A. Pavlik, B. Strohmaier, S. Tagesen, and J. Martinez-Rico, Physics Data 13-5, 34 (1990), in [87].

[30] C. M. Baglin, Nucl. Data Sheets 91, 423 (2000).

[31] M. Wagner, Report INDC(AUS)-D14, Vienna (1991), in [87].

[32] D. L. Smith, A. J. M. Plompen, and V. Semkova, Correction for low energy neutrons by spectral indexing, International Evaluation Co-operation, Vol. 19, Neutron activation crosssection measurements from threshold to $20 \mathrm{MeV}$ for the validation of nuclear models and their parameters, A. J. M. Plompen, co-ordinator, Nuclear Energy Agency, OECD, Issy-les-Moulineaux, France (2005).

[33] R. Dóczi, S. Sudár, J. Csikai, and S. M. Qaim, Phys. Rev. C 58, 2577 (1998).

[34] P. Reimer, A. J. Koning, A. J. M. Plompen, S. M. Qaim, and S. Sudár, Nucl. Phys. A815, 1 (2009).

[35] J. F. Briesmeister, MCNP ${ }^{\mathrm{TM}}$ - A General Monte Carlo N-Particle Transport Code, Report LA-13709-M, Los Alamos National Laboratory, Los Alamos NM, USA (2000).

[36] A. J. Koning and M. C. Duijvestijn, Nucl. Instrum. Methods Phys. Res. B 248, 197 (2006).

[37] M. Uhl and B. Strohmaier, Computer code for particle induced activation cross section and related quantities, IRK Report 76/01, Vienna (1976).

[38] IAEA-NDS, Handbook for calculations of nuclear reaction data: RIPL-2, IAEA, Vienna, Austria, available online at wwwnds.iaea.org/RIPL-2 (2006).

[39] I. G. Birn, B. Strohmaier, H. Freiesleben, and S. M. Qaim, Phys. Rev. C 52, 2546 (1995).

[40] B. Strohmaier, M. Fassbender, and S. M. Qaim, Phys. Rev. C 56, 2654 (1997).

[41] C. D. Nesaraja, S. Sudár, and S. M. Qaim, Phys. Rev. C 68, 024603 (2003).

[42] A. J. Koning, S. Hilaire, and M. C. Duijvestijn, TALYS: Comprehensive nuclear reaction modeling, in Proceedings of the International Conference on Nuclear Data for Science and Technology-ND2004, September 26-October 1, 2004, Santa Fe, USA, AIP Conf. Proc. No. 769 (Melville, NY, 2005), p. 1154.

[43] A. J. Koning and J. P. Delaroche, Nucl. Phys. A713, 231 (2003).

[44] A. J. Koning, M. C. Duijvestijn, S. C. van der Marck, R. Klein Meulekamp, and A. Hogenbirk, Nucl. Sci. Eng. 156, 357 (2007).

[45] A. J. Koning, S. Hilaire, and S. Goriely, Nucl. Phys. A810, 13 (2008).

[46] A. J. Koning and M. C. Duijvestijn, Nucl. Phys. A744, 15 (2004).

[47] J. Kopecky and M. Uhl, Phys. Rev. C 41, 1941 (1990).

[48] D. M. Brink, Nucl. Phys. 4, 215 (1957).

[49] P. Axel, Phys. Rev. 126, 671 (1962).

[50] D. G. Gardner, Neutron Radiative Capture, OECD/NEA Series on Neutron Physics and Nuclear Data in Science and Technology, edited by A. Michaudon et al. (1984).

[51] M. R. Bhat, Evaluated Nuclear Structure Data File, www.nndc. bnl.gov, NNDC, Brookhaven, USA (2003).
[52] E. Bauge, J. P. Delaroche, and M. Girod, Phys. Rev. C 58, 1118 (1998).

[53] E. Bauge, J. P. Delaroche, and M. Girod, Phys. Rev. C 63, 024607 (2001)

[54] S. Hilaire and S. Goriely, Nucl. Phys. A779, 63 (2006).

[55] O. Bersillon, SCAT-2: Un programme de modèle optique sphérique, Note CEA-N-2227, CEN-Bruyeres-le-Chatel (1981).

[56] J. C. Ferrer, J. D. Carlson, and J. Rapaport, Nucl. Phys. A275, 325 (1977).

[57] L. McFadden and G. R. Satchler, Nucl. Phys. A84, 177 (1966).

[58] W. Dilg, W. Schantl, H. Vonach, and M. Uhl, Nucl. Phys. A217, 269 (1973).

[59] V. I. Plyaskin and R. A. Kosilov, Level Density Parameters for the Back-Shifted Fermi Gas Model in the Mass Range $24 \leqslant A \leqslant 250$, Report INDC(CCP)-424 (IAEA, Vienna, Austria, 2000).

[60] R. Dóczi, S. Sudár, A. Fenyvesi, S. Szegedi, M. Fayez-Hassan, S. M. Qaim, and J. Csikai, Validation of neutron reference activation libraries, in Proceedings of the Workshop of Subgroup 19 on Activation Cross Sections, Final Report, Part B, OECDNEA, NSC-WPEC, Issy-les-Moulineaux, France (2005), p. 111.

[61] S. Begun, I. Kadenko, V. Maidanyuk, V. Neplyuev, G. Primenko, and V. Tarankanov, J. Nucl. Sci. Technol. S2, 425 (2002).

[62] T. B. Ryves, P. Kolkowski, and A. C. Hooley, Ann. Nucl. Energy 17, 107 (1990).

[63] G. Maslov, F. Nasyrov, and N. Pashkin, Yad. Konst. 9, 50 (1972).

[64] E. B. Paul and R. L. Clarke, Can. J. Phys. 31, 267 (1953).

[65] A. K. Hankla and R. W. Fink, Nucl. Phys. A180, 157 (1972).

[66] G. E. Belovickij, O. S. Presnjak, and L. V. Sukhov, in Proceedings of the 3rd All Union Conference on Neutron Physics, Kiev, USSR, 9-13 June 1975 (1976), p. 76.

[67] N. Lakshmana Das, C. V. Srinivasa Rao, and J. Rama Rao, Ann. Nucl. Energy 8, 283 (1981).

[68] R. Bass and R. Wechsung, Report EANDC(E)-89, EXFOR entry 21504 [88] (1968).

[69] P. Welch, J. Johnson, G. Randers-Pehrson, and J. Rapaport, Bull. Am. Phys. Soc. 26, 708 (1981), EXFOR entry 12912 [88].

[70] A. A. Filatenkov, S. V. Chuvaev, V. N. Aksenov, V. A. Yakovlev, A. V. Malyshenkov, S. K. Vasil'ev, M. Avrigeanu, V. Avrigeanu, D. L. Smith, Y. Ikeda et al., Systematic Measurement of Activation Cross Sections at Neutron Energies from 13.4 to 14.9 MeV, IAEA Report INDC(CCP)-402, Vienna, 1997; Khlopin Radium Institute Report RI-252, St. Petersburg (1999).

[71] A. Grallert, J. Csikai, C. M. Buczko, and I. Shaddad, Technical Report 286, INDC(NDS) (1993).

[72] Y. Yu, Nucl. Phys. A98, 451 (1967).

[73] D. L. Smith and J. W. Meadows, Neutron inelastic scattering studies for Lead 204, Report ANL-NDM-37 (1977).

[74] P. Bornemisza-Paupertl, J. Karolyi, and G. Peto, ATOMKI Kozlemenyek 10, 112 (1968), EXFOR entry 30388, [88].

[75] J. Csikai and G. Peto, Acta Physica Hungarica 23, 87 (1967).

[76] I. Kimura and K. Kobayashi, Nucl. Sci. Eng. 106, 332 (1990).

[77] P. Decowski, W. Grochulski, J. Karolyi, A. Marcinkowski, J. Piotrowski, E. Saad, and Z. Wilhelmi, Nucl. Phys. A204, 121 (1973).

[78] L. Hanlin, Z. Wenrong, Y. Weixiang, and Y. Xialin, Chinese J. Nucl. Phys. 12, 269 (1990).

[79] A. A. Druzhinin, N. I. Ivanova, and A. A. Lbov, Yad. Fiz. 14, 682 (1971). 
[80] W. Dilg, H. Vonach, G. Winkler, and P. Hille, Nucl. Phys. A118, 9 (1968).

[81] Y. Ikeda, C. Konno, K. Oishi, T. Nakamura, H. Miyade, K. Kawade, H. Yamamoto, and T. Katoh, Activation Cross Section Measurements for Fusion Reactor Structural Materials at Energies from 13.3 to $15.0 \mathrm{MeV}$ using FNS Facility, JAERI-1312 (1988).

[82] J. Karolyi, J. Csikai, and G. Peto, Nucl. Phys. A122, 234 (1968).

[83] M. Bormann, C. Abels, W. Carstens, and I. Riehle, Excitation functions of some fast neutron reactions, Report EANDC(E)-76, p. 51, EXFOR entry 21503 [88] (1967).

[84] G. Peto, P. Bornemisza-Pauspertl, and J. Karolyi, Acta Phys. Acad. Sci. Hung. 25, 91 (1968).
[85] E. Rurarz, J. Chwaszczewska, Z. Haratym, M. Pietrzykowski, and A. Sulik, Acta Phys. Pol. B 2, 553 (1971).

[86] R. Pepelnik, B. Anders, and B. M. Bahal, in Proceedings of the International Conference on Nuclear Data for Basic and Applied Science, edited by P. G. Young, R. E. Brown, G. F. Auchampaugh, P. W. Lisowski, and L. Stewart (Gordon and Breach Science Publishers, Santa Fe, NM, 1985), p. 211.

[87] H. Condé, Nuclear Data Standards for Nuclear Measurements, Report NEANDC-311 “U”, OECD-Nuclear Energy Agency, Paris, France (1992).

[88] EXFOR, Nuclear reaction data, www.nndc.bnl.gov (CSISRS), www.nea.fr/html/dbdata/x4/x4ret.html or www-nds.iaea.or.at/ exfor (2006). 